\title{
Morphokinetic parameters as a source of information concerning embryo developmental and implantation potential
}

\author{
Robert Milewski ${ }^{1}$, Jan Czerniecki², Agnieszka Kuczyńska3, ${ }^{3}$, Bożena Stankiewicz ${ }^{3}$, \\ Waldemar Kuczyński ${ }^{3,5}$ \\ ${ }^{1}$ Department of Statistics and Medical Informatics, Medical University of Bialystok, Poland \\ ${ }^{2}$ Department of Biology and Pathology of Human Reproduction, Institute of Animal Reproduction and Food Research, \\ Polish Academy of Sciences, Olsztyn, Poland \\ ${ }^{3}$ Centre for Reproductive Medicine KRIOBANK, Bialystok, Poland \\ ${ }^{4}$ Department of Reproduction and Gynecological Endocrinology, Medical University of Bialystok, Poland \\ ${ }^{5}$ Department of Gynecology, Medical University of Bialystok, Poland
}

\begin{abstract}
Objectives: The aim of the study was to present the results of time-lapse observation and to verify whether morphokinetic parameters are associated with embryo developmental and implantation potential.

Material and methods: The analysed data concern the development of 1,060 embryos, 898 of which (84.72\%) achieved the blastocyst stage and 307 were transferred into the uterine cavity. As a result, 126 (41.04\%) biochemical pregnancies and 109 (35.50\%) clinical pregnancies were observed. Time from fertilisation to further divisions into 2-9 blastomeres, first to fourth round of cleavage, second to third synchronisation parameters and the duration of stages after the first, second and third division were analysed.

Results: Most of the parameters in the group of embryos developed to the blastocyst stage reached lower values than in the non-developed group. Moreover, parameters in the first group clearly had less dispersion. The differences between the groups with and without a biochemical pregnancy were smaller than the differences in the analysis of development to the blastocyst stage. However, in the case of clinical pregnancy analysis, there were again larger differences between both groups. A strong correlation was found between the majority of absolute morphokinetic parameters. A weaker, but still statistically significant correlation, was established between relative and other parameters.

Conclusions: Morphokinetic parameters are associated with embryo developmental and implantation potential and can be considered as predictors of their quality. However, the development of efficient pregnancy prediction models needs further research utilising information from all available parameters and using advanced biostatistical methods.
\end{abstract}

Key words: infertility, IVF ET, morphokinetic parameters, Embryoscope, time-lapse

Ginekologia Polska 2016; 87, 10: 677-684

\section{INTRODUCTION}

It is estimated that in today's society more than $10 \%$ of couples suffer from infertility [1]. Social, genetic and environmental factors are responsible for this situation. For some couples facing infertility the only real possibility of conception and a successful delivery is provided by assisted reproductive techniques (ART). More than three decades have passed since the birth of the first child conceived using the in vitro fertilisation (IVF) method. Tremendous progress has been made regarding this method, the protocols and media composition, which has brought a vast improvement in the effectiveness of the treatment. Currently, the success rate of ART stands at approximately $40 \%$, which means that it is higher than the success rate of natural conception 
[2]. Despite the progress and wide availability of infertility treatment using IVF many issues remain unresolved and unexplained. Consequently, further improvements to increase treatment efficacy and effectiveness are difficult to achieve. One of the key problems is the difficulty with the precise evaluation of the developmental potential of embryos obtained after in vitro fertilisation. Therefore, embryos of unknown implantation potential are frequently transferred. Pregnancy rates can be significantly improved by transferring a greater number of embryos, but this may produce an undesirable increase in the incidence of multiple pregnancies and related gynecological complications.

It is therefore crucial to develop new, non-invasive biomarkers of embryo developmental potential other than those based on morphological criteria. This would increase the effectiveness of IVF treatment using only single embryo transfers. Recent reports indicate that time-lapse imaging can play an important role in this process [3]. This technique offers the possibility of continuous observation of embryo development, providing a new method for more sensitive and precise data collection. Associations between specific morphokinetic events and embryo developmental and implantation potential have been identified, suggesting that this information may improve embryo selection for transfer [4].

\section{OBJECTIVES}

The aim of this study was to present the results of time-lapse observation and to verify whether morphokinetic parameters are associated with embryo developmental and implantation potential.

\section{MATERIAL AND METHODS}

Types of morphokinetic parameters are presented in Table 1. They are divided into two groups: absolute - those which measure the time from insemination ( $\mathrm{t} 0$ ) and relative - the distance between two time-points in the embryo development. The latter have their biological interpretation (for example duration of the $n$-th round of cleavage or difference between the first and the last cleavage in a round) $[5,6]$.

A retrospective study was carried out using data collected in the Centre for Reproductive Medicine Kriobank in Bialystok, Poland, between June 2012 and April 2015. Embryos obtained from oocytes collected after stimulation of multiple ovulation were fertilised by the intracytoplasmic sperm injection (ICSI) and placed in one of the twelve wells of a special culture dish for the time-lapse process, each containing a culture medium droplet of $20 \mu$ l of Quinn's Advantage Protein Plus Cleavage Medium (SAGE, USA). The drops were covered with mineral oil (SAGE, USA) in order to prevent changes in fluid osmolarity. The embryos were incubated at the temperature of $37.0^{\circ} \mathrm{C}, \mathrm{CO}_{2}$ concentration of $5.0 \%$ and decreased $\mathrm{O}_{2}$ concentration of $5.0 \%$. Images

\begin{tabular}{|c|c|c|}
\hline Parameter & Formula & Definition \\
\hline to & & time of insemination \\
\hline tPB2 & & $\begin{array}{l}\text { time from insemination to appearance } \\
\text { of second polar body }\end{array}$ \\
\hline $\mathrm{tPNa}$ & & $\begin{array}{l}\text { time from insemination to pronuclei } \\
\text { appearance }\end{array}$ \\
\hline tPNf & & time from insemination to pronuclei fading \\
\hline t2-t9 & & $\begin{array}{l}\text { times from insemination to corresponding } \\
\text { divisions (two to nine) }\end{array}$ \\
\hline tM & & $\begin{array}{l}\text { time from insemination to compacting } \\
\text { into the morula stage }\end{array}$ \\
\hline $\mathrm{tSB}$ & & $\begin{array}{l}\text { time from insemination to start of } \\
\text { blastulation }\end{array}$ \\
\hline $\mathrm{tB}$ & & $\begin{array}{l}\text { time from insemination to blastocyst } \\
\text { formation complete }\end{array}$ \\
\hline tEB & & $\begin{array}{l}\text { time from insemination to expanded } \\
\text { blastocyst }\end{array}$ \\
\hline $\mathrm{tHB}$ & & $\begin{array}{l}\text { time from insemination to hatched } \\
\text { blastocyst }\end{array}$ \\
\hline cc1 & t2-t0 & the first round of cleavage, equal to $t 2$ \\
\hline $\mathrm{cc} 2$ & t3-t2 & the second round of cleavage \\
\hline $\mathrm{cc} 3$ & t5-t3 & the third round of cleavage \\
\hline cc4 & t9-t5 & the fourth round of cleavage \\
\hline s1 & $\mathrm{t} 2-\mathrm{t} 2$ & $\begin{array}{l}\text { the first synchronisation parameter, always } \\
\text { equal to zero, parameter not used }\end{array}$ \\
\hline s2 & t4-t3 & the second synchronisation parameter \\
\hline s3 & t8-t5 & the third synchronisation parameter \\
\hline t2_int & t3-t2 & the stage after first division, equal to cc2 \\
\hline t4_int & $\mathrm{t} 5-\mathrm{t} 4$ & the stage after second division \\
\hline t8_int & t9-t8 & the stage after third division \\
\hline
\end{tabular}

of each embryo were acquired every 7 minutes at five different focal planes.

The data concerning the development of 1,060 embryos to stage t 9 were analysed. Out of the total number of embryos, 898 (84.72\%) achieved the blastocyst stage, from which 307 were transferred into the uterine cavity. Only embryos from the single embryo transfer (SET) or embryos from the double transfer with no pregnancy were included in the analysis. Cases of a double transfer with a singular pregnancy were excluded from analysis due to the fact that it is impossible to identify which embryo implanted. As a result, 126 (41.04\%) biochemical pregnancies were observed, 109 (35.50\%) of which developed into clinical pregnancies. We analysed parameters $t 2$ to $t 9$, the first to fourth round of cleavage, the second to third synchronization parameters and the duration of stages after the first, second and third division.

All absolute morphokinetic parameters and a majority of relative parameters are non-monotonic since very low 
Table 2. Medians in the "positive outcome" groups

\begin{tabular}{|c|c|c|c|}
\hline Parameter & $\begin{array}{c}\text { Development to the blastocyst stage } \\
(\mathbf{n}=\mathbf{8 9 8})\end{array}$ & $\begin{array}{c}\text { Biochemical pregnancy } \\
(\mathbf{n}=\mathbf{1 2 6})\end{array}$ & $\begin{array}{c}\text { Clinical pregnancy } \\
(\mathbf{n}=\mathbf{1 0 9})\end{array}$ \\
\hline t2 & 26.70 & 26.51 & 26.56 \\
\hline t3 & 37.58 & 36.90 & 36.94 \\
\hline t4 & 38.89 & 38.67 & 38.77 \\
\hline t5 & 51.64 & 50.67 & 50.85 \\
\hline t6 & 52.97 & 51.38 & 51.39 \\
\hline t7 & 55.06 & 53.74 & 53.67 \\
\hline t8 & 60.43 & 58.10 & 58.25 \\
\hline t9 & 76.16 & 75.22 & 75.21 \\
\hline cc2 & 10.96 & 10.89 & 10.98 \\
\hline cc3 & 13.73 & 13.43 & 13.43 \\
\hline cc4 & 24.03 & 23.87 & 23.70 \\
\hline t4_int & 12.49 & 12.32 & 12.35 \\
\hline t8_int & 14.96 & 16.25 & 16.36 \\
\hline
\end{tabular}

as well as very high values are not accurate predictors of embryo developmental potential. Therefore, a direct comparison of their values using statistical difference tests is not useful. A suitable transformation which can convert non-monotonic parameters into their monotonic forms is needed for further analysis. Milewski et al. presented a hypothesis that the values of the parameter most favourable to the highest reproductive potential will be close to the median value in the "positive outcome" group of embryos $[7,8]$. Therefore, the median value in the groups of embryos developed to the blastocyst stage, a biochemical and a clinical pregnancy was estimated (Tab. 2).

Following that, the non-monotonic parameters were transformed by determining their distance from the median:

$$
t_{\text {med }}=|t-\operatorname{Median}(t)|
$$

which enabled the performance of a comparative analysis between groups determined on the basis of the development to the blastocyst stage, a biochemical and a clinical pregnancy. Since only synchronisation parameters are monotonic (the zero value means full synchronisation of divisions, a higher value means greater asynchrony), they do not need any transformation.

Statistical analysis of the normality distribution was verified by the Kolmogorov-Smirnov test with the Lilliefors' amendment and the Shapiro-Wilk test. The quantitative variables did not follow normal distribution. The non-parametric U Mann-Whitney test was used to compare the values of the analysed parameters between the two groups. The Spearman rank-order correlation coefficient was determined in order to estimate the correlation between the analysed parameters. Statistical significance was determined at the $\mathrm{p}<0.05$ level. The Statistica 12.0 software (StatSoft, Tulsa, OK, USA) was used in statistical analysis.

\section{RESULTS}

Distributions of absolute morphokinetic parameters in the groups of embryos developed and non-developed to the blastocyst stage are shown in Figure 1. Most of the parameters in the group of embryos developed to the blastocyst stage have lower values than those in the group of embryos which did not reach this stage. Furthermore, the parameters in the first group clearly show a smaller dispersion.

Figure 2 shows the distribution of absolute morphokinetic parameters with respect to biochemical pregnancy. The differences between both groups are not as considerable as those shown in the analysis of embryo development to the blastocyst stage. Lower values of parameters in the biochemical pregnancy group are evident only for parameters $\mathrm{t} 5$ and higher. Moreover, differences in parameter

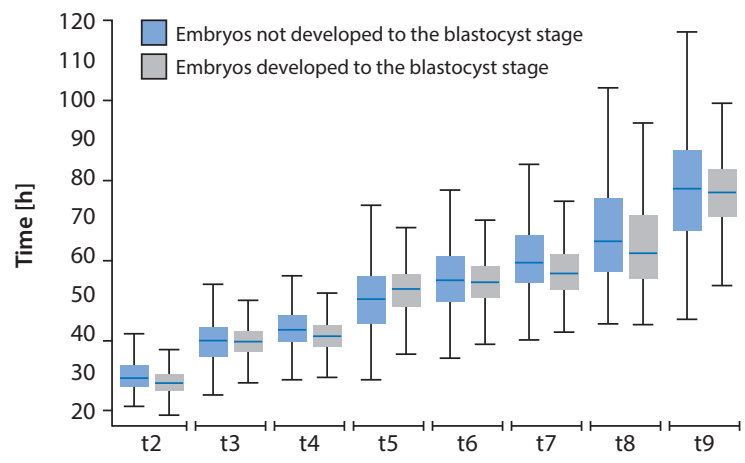

Figure 1. Distribution of morphokinetic parameters for embryos developed and not developed to the blastocyst stage (median, quartiles and non-outlier range) 


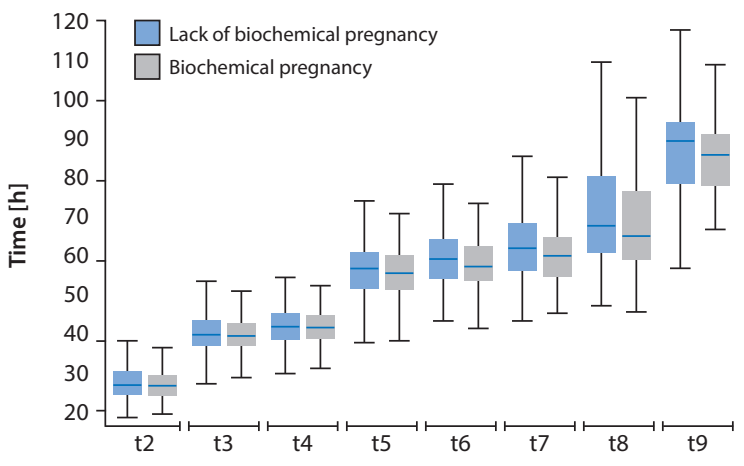

Figure 2. Distribution of morphokinetic parameters with respect to biochemical pregnancy (median, quartiles and non-outlier range)

dispersion between the two groups are much lower than in the analysis of embryos developed and non-developed to the blastocyst stage. Minimal differences are observed only for the last parameters.

A similar observation was made in the clinical pregnancy analysis. However, marginally larger differences in parameter distribution were observed between both groups in comparison to a biochemical pregnancy (Fig. 3).

The morphokinetic parameters transformed into the form of a distance from a median ( $s 2$ and $s 3$ did not require transformation) were compared in terms of the development to the blastocyst stage (Tab. 3). In all cases, the values in the group of embryos developed to the blastocyst stage were closer to zero in comparison with the group of undeveloped embryos. Moreover, the differences between both groups increased with

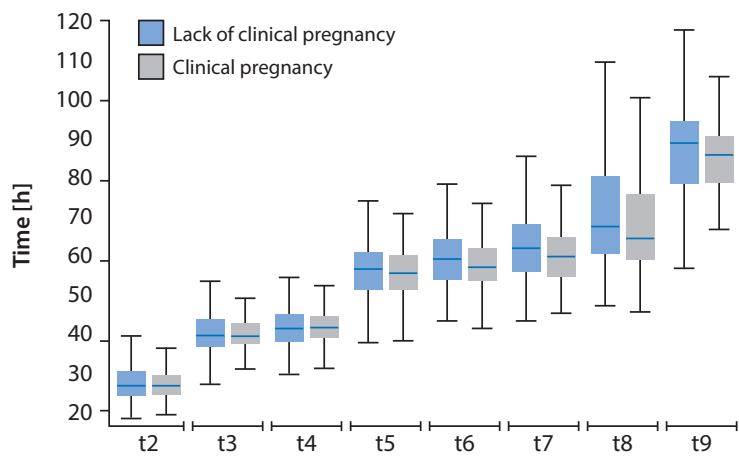

Figure 3. Distribution of morphokinetic parameters with respect to clinical pregnancy (median, quartiles and non-outlier range)

time. The difference between the values of $\mathrm{t} 2_{\text {med }}$ parameter was not yet statistically significant, but for other parameters ( $\mathrm{t} 3_{\text {med }}$ upwards) the differences became significant, achieving a greater significance for consecutive parameters. The medians of the $t 9_{\text {med }}$ parameter were as follows: in the group of embryos developed to the blastocysts stage $\mathrm{Me}=5.62(\mathrm{Q} 1=2.65$, $\mathrm{Q} 3=10.01$ ) and in the group of embryos that did not develop to the blastocyst stage: $\mathrm{Me}=10.31(\mathrm{Q} 1=5.04, \mathrm{Q} 3=16.91)$. The median value for the second group of embryos was up to 83.5\% higher than in the first group.

The comparison of parameters between the groups with and without a biochemical pregnancy revealed differences which were far less statistically significant (Tab. 4). Although in most cases values in the biochemical pregnancy group were lower than in the group without pregnancy, statistically

Table 3. Comparison of embryos developed and not developed to the blastocyst stage

\begin{tabular}{|c|c|c|c|c|c|c|c|c|c|c|c|}
\hline \multirow{2}{*}{ Parameter } & \multicolumn{5}{|c|}{$\begin{array}{l}\text { Embryos developed to the blastocyst stage } \\
\qquad(n=898)\end{array}$} & \multicolumn{5}{|c|}{$\begin{array}{l}\text { Embryos not developed to the blastocyst stage } \\
\qquad(n=162)\end{array}$} & \multirow[t]{2}{*}{ p-value } \\
\hline & Min & $Q_{1}$ & Me & $Q_{3}$ & Max & Min & $Q_{1}$ & Me & $Q_{3}$ & Max & \\
\hline $\mathrm{t} 2_{\text {med }}$ & 0.00 & 1.02 & 2.04 & 3.59 & 77.98 & 0.03 & 1.00 & 2.29 & 4.06 & 76.93 & 0.27 \\
\hline $\mathrm{t} 3_{\text {med }}$ & 0.01 & 1.31 & 2.60 & 4.36 & 77.82 & 0.03 & 1.33 & 2.85 & 6.11 & 76.45 & 0.04 \\
\hline t4 $4_{\text {med }}$ & 0.01 & 1.39 & 2.67 & 4.56 & 77.33 & 0.02 & 1.39 & 3.13 & 6.12 & 75.96 & 0.02 \\
\hline t5 $5_{\text {med }}$ & 0.01 & 1.78 & 3.78 & 6.35 & 77.89 & 0.06 & 2.04 & 4.33 & 10.45 & 64.92 & 0.0007 \\
\hline$t 6_{\text {med }}$ & 0.00 & 1.93 & 3.95 & 6.39 & 77.74 & 0.03 & 2.21 & 5.08 & 10.20 & 79.73 & 0.0002 \\
\hline $\mathrm{t} 7_{\text {med }}$ & 0.01 & 2.06 & 4.61 & 7.98 & 76.23 & 0.03 & 2.52 & 6.47 & 12.85 & 78.22 & 0.0002 \\
\hline $\mathrm{t} 8_{\text {med }}$ & 0.05 & 3.73 & 7.63 & 12.26 & 71.45 & 0.08 & 4.55 & 10.42 & 18.22 & 76.24 & 0.0001 \\
\hline t9 ${ }_{\text {med }}$ & 0.01 & 2.65 & 5.62 & 10.01 & 77.62 & 0.15 & 5.04 & 10.31 & 16.91 & 88.86 & $<0.0001$ \\
\hline $\mathrm{CC}{ }_{\text {med }}$ & 0.01 & 0.48 & 0.95 & 1.70 & 10.37 & 0.01 & 0.66 & 1.46 & 2.82 & 17.14 & $<0.0001$ \\
\hline $\mathrm{CC}_{\text {med }}$ & 0.01 & 0.70 & 1.49 & 2.62 & 19.49 & 0.01 & 0.88 & 2.18 & 4.49 & 18.14 & $<0.0001$ \\
\hline $\mathrm{CC}_{\text {med }}$ & 0.03 & 1.50 & 3.18 & 5.92 & 26.01 & 0.21 & 3.38 & 6.29 & 13.05 & 36.21 & $<0.0001$ \\
\hline s2 & 0.00 & 0.47 & 0.94 & 1.75 & 17.91 & 0.00 & 0.35 & 1.06 & 1.99 & 12.08 & 0.85 \\
\hline s3 & 0.00 & 3.28 & 7.18 & 19.03 & 42.45 & 0.35 & 4.79 & 14.08 & 23.34 & 52.41 & $<0.0001$ \\
\hline t4int $t_{\text {med }}$ & 0.00 & 0.75 & 1.58 & 2.98 & 15.85 & 0.05 & 1.25 & 2.46 & 6.25 & 18.08 & $<0.0001$ \\
\hline tsint $_{\text {med }}$ & 0.01 & 3.40 & 6.48 & 10.76 & 16.43 & 0.06 & 3.66 & 7.83 & 11.45 & 22.38 & 0.15 \\
\hline
\end{tabular}




\begin{tabular}{|c|c|c|c|c|c|c|c|c|c|c|c|}
\hline \multirow[t]{2}{*}{ Parameter } & \multicolumn{5}{|c|}{$\begin{array}{l}\text { Biochemical pregnancy } \\
\qquad(n=126)\end{array}$} & \multicolumn{5}{|c|}{$\begin{array}{l}\text { Lack of biochemical pregnancy } \\
\qquad(n=181)\end{array}$} & \multirow[t]{2}{*}{ p-value } \\
\hline & Min & $Q_{1}$ & Me & $Q_{3}$ & Max & Min & $Q_{1}$ & $\mathrm{Me}$ & $Q_{3}$ & Max & \\
\hline $\mathrm{t} 2_{\text {med }}$ & 0.01 & 0.98 & 2.05 & 3.19 & 78.18 & 0.03 & 1.09 & 2.24 & 3.64 & 10.90 & 0.46 \\
\hline $\mathrm{t} 3_{\text {med }}$ & 0.01 & 1.16 & 2.32 & 3.91 & 78.51 & 0.01 & 1.27 & 2.49 & 4.52 & 13.34 & 0.24 \\
\hline $\mathrm{t} 4_{\text {med }}$ & 0.03 & 1.09 & 2.36 & 4.34 & 77.55 & 0.06 & 1.46 & 2.70 & 4.26 & 14.49 & 0.17 \\
\hline $\mathrm{t} 5_{\text {med }}$ & 0.19 & 1.68 & 3.62 & 4.96 & 78.87 & 0.10 & 2.12 & 3.57 & 5.94 & 23.55 & 0.24 \\
\hline $\mathrm{t} 6_{\text {med }}$ & 0.02 & 1.62 & 3.78 & 5.41 & 79.34 & 0.05 & 1.96 & 3.97 & 6.56 & 27.02 & 0.08 \\
\hline $\mathrm{t} 7_{\text {med }}$ & 0.07 & 2.03 & 4.23 & 6.34 & 77.56 & 0.02 & 2.33 & 4.31 & 7.71 & 27.49 & 0.33 \\
\hline $\mathrm{t} 8_{\text {med }}$ & 0.15 & 3.27 & 6.73 & 10.45 & 73.78 & 0.01 & 2.80 & 6.20 & 12.30 & 40.60 & 0.91 \\
\hline $\mathrm{t} 9_{\text {med }}$ & 0.01 & 2.25 & 4.85 & 8.44 & 78.56 & 0.10 & 3.38 & 6.19 & 10.59 & 41.36 & 0.006 \\
\hline $\mathrm{cc}_{\text {med }}$ & 0.01 & 0.45 & 1.03 & 1.61 & 9.03 & 0.02 & 0.45 & 0.91 & 1.77 & 10.30 & 0.99 \\
\hline $\mathrm{CC}{ }_{\text {med }}$ & 0.00 & 0.47 & 1.05 & 2.21 & 11.30 & 0.00 & 0.59 & 1.57 & 2.53 & 12.44 & 0.08 \\
\hline $\mathrm{CC} 4_{\text {med }}$ & 0.01 & 1.01 & 2.62 & 5.05 & 25.55 & 0.05 & 1.94 & 3.74 & 6.80 & 36.44 & 0.0003 \\
\hline s2 & 0.00 & 0.58 & 0.94 & 1.75 & 10.39 & 0.00 & 0.58 & 0.94 & 1.75 & 14.64 & 0.78 \\
\hline s3 & 0.00 & 3.06 & 5.17 & 17.42 & 27.28 & 0.00 & 3.30 & 7.47 & 18.58 & 41.15 & 0.14 \\
\hline $\mathrm{t}_{\text {4int }}{ }_{\text {med }}$ & 0.03 & 0.65 & 1.32 & 2.64 & 11.95 & 0.01 & 0.68 & 1.58 & 2.91 & 11.24 & 0.19 \\
\hline t8int $_{\text {med }}$ & 0.08 & 2.78 & 4.57 & 8.30 & 15.42 & 0.02 & 3.43 & 7.01 & 11.68 & 16.27 & 0.007 \\
\hline
\end{tabular}

\begin{tabular}{|c|c|c|c|c|c|c|c|c|c|c|c|}
\hline \multirow{2}{*}{ Parameter } & \multicolumn{5}{|c|}{$\begin{array}{l}\text { Clinical pregnancy } \\
\qquad(n=109)\end{array}$} & \multicolumn{5}{|c|}{$\begin{array}{l}\text { Lack of clinical pregnancy } \\
\qquad(n=198)\end{array}$} & \multirow[t]{2}{*}{ p-value } \\
\hline & Min & $Q_{1}$ & Me & $\mathbf{Q}_{3}$ & Max & Min & $Q_{1}$ & Me & $Q_{3}$ & Max & \\
\hline $\mathrm{t} 2_{\text {med }}$ & 0.00 & 0.92 & 1.98 & 3.18 & 78.12 & 0.01 & 1.12 & 2.23 & 3.62 & 10.84 & 0.25 \\
\hline $\mathrm{t} 3_{\text {med }}$ & 0.00 & 1.08 & 1.99 & 3.56 & 78.46 & 0.02 & 1.31 & 2.53 & 4.54 & 13.38 & 0.07 \\
\hline $\mathrm{t} 4_{\text {med }}$ & 0.00 & 1.10 & 2.24 & 3.96 & 77.45 & 0.00 & 1.49 & 2.77 & 4.46 & 14.39 & 0.05 \\
\hline $\mathrm{t} 5_{\text {med }}$ & 0.00 & 1.65 & 3.55 & 4.75 & 78.68 & 0.02 & 2.31 & 3.55 & 6.18 & 23.36 & 0.09 \\
\hline t6 $6_{\text {med }}$ & 0.00 & 1.59 & 3.65 & 5.01 & 79.32 & 0.06 & 1.94 & 4.04 & 6.54 & 27.00 & 0.03 \\
\hline $\mathrm{t} 7_{\text {med }}$ & 0.00 & 1.94 & 4.21 & 6.29 & 77.62 & 0.05 & 2.39 & 4.25 & 7.64 & 27.55 & 0.29 \\
\hline t8 ${ }_{\text {med }}$ & 0.00 & 3.42 & 6.74 & 9.96 & 73.63 & 0.08 & 2.76 & 6.43 & 12.35 & 40.45 & 0.70 \\
\hline t9 ${ }_{\text {med }}$ & 0.00 & 2.21 & 4.74 & 8.08 & 78.56 & 0.10 & 3.33 & 6.37 & 10.43 & 41.36 & 0.005 \\
\hline $\mathrm{CC}{ }_{\text {med }}$ & 0.00 & 0.46 & 0.93 & 1.52 & 8.64 & 0.00 & 0.46 & 0.82 & 1.78 & 10.39 & 0.71 \\
\hline $\mathrm{Cc}{ }_{\text {med }}$ & 0.00 & 0.46 & 1.01 & 1.93 & 11.30 & 0.00 & 0.59 & 1.57 & 2.64 & 12.44 & 0.02 \\
\hline $\mathrm{CC}_{\text {med }}$ & 0.00 & 1.05 & 2.57 & 4.88 & 25.71 & 0.11 & 1.95 & 3.84 & 6.76 & 36.60 & 0.0005 \\
\hline s2 & 0.00 & 0.58 & 0.93 & 1.75 & 10.39 & 0.00 & 0.58 & 0.94 & 1.75 & 14.64 & 0.88 \\
\hline s3 & 0.00 & 3.04 & 5.14 & 16.82 & 27.28 & 0.00 & 3.38 & 7.48 & 18.69 & 41.15 & 0.09 \\
\hline $\mathrm{t}_{\text {int }}{ }_{\text {med }}$ & 0.00 & 0.63 & 1.27 & 2.47 & 11.92 & 0.04 & 0.68 & 1.61 & 2.98 & 11.27 & 0.06 \\
\hline t8int $_{\text {med }}$ & 0.00 & 2.66 & 4.43 & 7.96 & 15.53 & 0.01 & 3.52 & 6.75 & 11.57 & 16.24 & 0.008 \\
\hline
\end{tabular}

significant differences concerned only the three parameters associated with subsequent divisions: $\mathrm{t} 9_{\text {med }}, \mathrm{cc} 4_{\text {med }}$ and t8int $_{\text {med }}$. The median values for these parameters in the group without a biochemical pregnancy were higher by $27.6 \%, 42.7 \%$ and $53.4 \%$, respectively.

The comparative analyses conducted between the groups with and without a clinical pregnancy confirmed the existence of statistically significant differences for the aforementioned three parameters, and additionally demonstrated significant differences for the subsequent three parameters: $\mathrm{t} 4_{\text {med}^{\prime}} \mathrm{t} 6_{\text {med' }^{\prime}} \mathrm{cc} 3_{\text {med }}$ (Tab. 5). Furthermore, the next four parameters approached the threshold of statistical significance, reaching $\mathrm{p}$-value below 0.1 level ( $\mathrm{t} 3 \mathrm{med}^{\prime}$ $\mathrm{t} 5_{\text {med }^{\prime}} \mathrm{s} 3$ and $\left.\mathrm{t} 4 \mathrm{int}_{\text {med }}\right)$. The biggest differences between the groups were found for the following parameters: $\mathrm{cc} 3{ }_{\text {med' }}$ t8int ${ }_{\text {med }}$ and $\mathrm{Cc}_{\text {med }}$ where the medians in the group without 
Table 6. Correlations between morphokinetic parameters (Spearman R coefficients)

\begin{tabular}{|c|c|c|c|c|c|c|c|c|c|c|c|c|c|c|}
\hline & ${ }^{t} 3_{\text {med }}$ & t4 $4_{\text {med }}$ & t5 ${ }_{\text {med }}$ & t $6_{\text {med }}$ & t7 $7_{\text {med }}$ & t8 $8_{\text {med }}$ & t $9_{\text {med }}$ & $\mathrm{CC}_{\text {med }}$ & $\mathrm{CC}_{\text {med }}$ & $\mathrm{cc} 4_{\text {med }}$ & s2 & s3 & t4int ${ }_{\text {med }}$ & tsint $_{\text {med }}$ \\
\hline $\mathrm{t} 2_{\text {med }}$ & 0.63 & 0.64 & 0.50 & 0.48 & 0.39 & 0.21 & 0.27 & 0.11 & 0.15 & NS & 0.08 & 0.07 & 0.09 & NS \\
\hline $\mathrm{t} 3_{\text {med }}$ & & 0.77 & 0.76 & 0.69 & 0.54 & 0.24 & 0.39 & 0.38 & 0.27 & 0.09 & 0.14 & 0.13 & 0.22 & 0.06 \\
\hline $\mathrm{t} 4_{\text {med }}$ & & & 0.66 & 0.64 & 0.59 & 0.33 & 0.37 & 0.31 & 0.23 & 0.10 & 0.14 & 0.12 & 0.16 & NS \\
\hline $\mathrm{t} 5_{\text {med }}$ & & & & 0.85 & 0.68 & 0.31 & 0.56 & 0.38 & 0.57 & 0.18 & 0.10 & 0.16 & 0.47 & 0.09 \\
\hline $\mathrm{t} 6_{\text {med }}$ & & & & & 0.80 & 0.41 & 0.54 & 0.35 & 0.52 & 0.17 & 0.11 & 0.22 & 0.40 & 0.10 \\
\hline $\mathrm{t} 7_{\text {med }}$ & & & & & & 0.58 & 0.52 & 0.28 & 0.44 & 0.20 & 0.12 & 0.31 & 0.33 & 0.13 \\
\hline $\mathrm{t} 8_{\text {med }}$ & & & & & & & 0.38 & 0.15 & 0.26 & 0.20 & NS & 0.58 & 0.20 & 0.35 \\
\hline $\mathrm{t} 9_{\text {med }}$ & & & & & & & & 0.28 & 0.49 & 0.55 & 0.11 & 0.20 & 0.42 & 0.18 \\
\hline$c c 2_{\text {med }}$ & & & & & & & & & 0.28 & 0.15 & 0.14 & 0.15 & 0.23 & 0.06 \\
\hline $\mathrm{cc} 3_{\text {med }}$ & & & & & & & & & & 0.28 & 0.10 & 0.22 & 0.72 & 0.10 \\
\hline $\mathrm{CC}_{\text {med }}$ & & & & & & & & & & & 0.10 & 0.23 & 0.28 & 0.20 \\
\hline s2 & & & & & & & & & & & & 0.15 & 0.13 & NS \\
\hline s3 & & & & & & & & & & & & & 0.24 & 0.47 \\
\hline t4int ${ }_{\text {med }}$ & & & & & & & & & & & & & & 0.13 \\
\hline
\end{tabular}

Weak correlation

Strong correlation

Moderate correlation

Very strong correlation

clinical pregnancy were higher by $55.4 \%, 52.4 \%$ and $49.4 \%$, respectively.

Correlation analysis was performed in order to check the multicollinearity of morphokinetic parameters. Table 6 contains Spearman correlation coefficients calculated for all pairs of the analysed parameters. Statistically significant correlations were found in almost all the cases. The correlations were not statistically significant only for five pairs, where parameters were related to distant points of time. The strongest correlations were found for phenomena which occurred at close time points. A strong or very strong correlation was established between the majority of absolute morphokinetic parameters. The strongest correlation was observed between parameters $\mathrm{t} 5_{\text {med }}$ and $\mathrm{t} 6_{\text {med }}$ and $\mathrm{t} 6_{\text {med }}$ and $t 7_{\text {med }}(R=0.85$ and $R=0.80$, respectively).

\section{DISCUSSION}

Time-lapse techniques have opened a new chapter in embryo quality assessment. Analyses of the early stages of embryo development have revealed a relationship between embryo quality, its developmental potential and morphokinetic parameters. The pattern of the exact times of the first few cleavages can be regarded as a predictor of blastocyst stage achievement. The best quality embryos behave in a similar way, which indicates that the sequence of events is highly regulated. Any aberration, such as a delay in the initial divisions or their asynchrony, indicates a decreased chance of an embryo's development to blastocyst [9].
Our data show that the timing of further divisions correlates more strongly with blastocyst development than the timing of the first divisions (Tab. 3). The differences in the first and second division times between groups of embryos which achieved and those which did not achieve the blastocyst stage are not statistically significant whereas all the remaining division times (third and subsequent) differ significantly between the groups. It appears that poor quality embryos cumulate the delay during their growth instead of restoring the proper pattern of morphokinetic events. This may indicate that the sequence of events is determined from the outset but abnormalities can occur at different stages of development.

A comprehensive analysis of a broad range of features, rather than an analysis of individual parameters, can provide more in-depth information concerning embryo quality and their developmental potential. Analysis of relative time ratios, time intervals and cleavage synchrony has permitted the development of highly accurate predictive models of blastocyst formation. Cetinkaya et al. proved in their study that relative timings - cleavage synchronicity from 4 to 8 cells as well as from 2 to 8 cells - have a higher power of prediction of blastocyst stage development than absolute time points [10]. Furthermore, they noted that values of the analysed parameters may depend on the conditions present in IVF units. Therefore, it is necessary to adjust the model to meet the clinic's specific conditions.

The identification of the best quality embryos with the highest blastocyst formation potential could allow the uter- 
ine transfer of embryos in the second or third day of their life, before they achieve the blastocyst stage [7]. Elimination of the prolonged in vitro culturing may be beneficial, e.g. for reducing the risk of potential pregnancy complications. However, as pregnancy is the ultimate indicator of infertility treatment success, the most important issue is to identify embryos with the highest implantation potential. A comparison of the early stages of development between implanted and not implanted embryos has revealed that some morphokinetic parameters can differentiate these groups. The implanted embryos are characterised by an earlier appearance of nuclei in the first blastomere after the first cleavage and nucleus appearance in the first two blastomeres is more synchronised [11].

Pregnancy may be predicted more accurately with the application of models which combine static evaluation of embryo morphology and dynamic morphokinetic parameters. Meseguer et al. presented such a model with a hierarchical structure [12]. The researchers proved that selecting embryos using time-lapse categorisation was more effective for predicting implantation than using merely morphological categorisation. According to the proposed model, the analysed morphokinetic parameters have an optimal range and both lower and higher values indicate decreased implantation potential. It does not relate only to cleavage synchronisation, where any asynchrony is an unwanted phenomenon. Unfortunately, the high quality of the suggested model was not confirmed by further studies but the concept put forward by Meseguer et al. has not been negated [13]. It has been suggested that the lower prediction power of the selection algorithm created by Meseguer et al. may be a result of differing conditions present in different IVF units.

A similar approach to implantation modelling was employed by Aguilar et al. [14]. The authors determined the optimal ranges of morphokinetic parameters and used the values outside of these ranges as indicators of reduced implantation potential. The proper timing of the second polar body extrusion, the first and second pronucleus appearance, abuttal and fading were linked to embryo implantation potential. The logistic regression method was utilised to create the predictor of implantation, but following the ROC analysis, the area under the ROC curve (AUC) was equal to only 0.605 .

The models presented above prove the usefulness of information included in time-lapse data in predicting pregnancy. However, there are some problems that may limit prediction accuracy. One of them is connected with the fact that transferred embryos are naturally limited only to those which are characterised by the most optimal values of morphokinetic parameters. Therefore, differences between them are smaller and the prediction process must be less effective in comparison to the prediction of development to the blastocyst stage. Our analysis indicates that later events differentiate implanted and non-implanted embryos more clearly. Statistically significant differences between groups of embryos with and without a biochemical pregnancy were found for $\mathrm{t} 9_{\text {med }^{\prime}} \mathrm{Cc} 4_{\text {med }}$ and t8int ${ }_{\text {med }}$ parameters (Tab. 4). The situation was slightly different when a clinical pregnancy was analysed. In that case, besides the three variables mentioned above, we found statistically significant differences for three other parameters: $\mathrm{t} 4_{\text {med }} \mathrm{t} 6_{\text {med }}$ and $\mathrm{cc} 3_{\text {med }}$ (Tab. 5). Moreover, parameters $\mathrm{t} 3_{\text {med' }} \mathrm{t} 5_{\text {med }^{\prime}} \mathrm{s} 3$ and $\mathrm{t} 4 \mathrm{int} \mathrm{med}_{\text {med }}$ showed differences at a level slightly above the threshold for statistical significance. A clinical pregnancy is reported later than a biochemical pregnancy, when more time has passed since implantation. During that time the interaction between the embryo and the endometrium may begin to exert'selection pressure', which may lead to the natural abortion of lower quality embryos. Only highest grade embryos can survive for a sufficiently long period following implantation to give the clinical symptoms of pregnancy. Therefore, differentiation criteria in this case are easier to observe.

All morphokinetic parameters are strongly correlated to one another (Tab. 6). This fact can be considered a technical problem because the majority of statistical methods (e.g. the logistic regression method) require an assumption that only uncorrelated, independent variables can be incorporated into the model. However, this problem can be combated with the use of advanced data mining techniques such as principal component analysis (PCA) which transforms correlated variables into uncorrelated principal components. They bring the same information as the primary variables and can be analysed using e.g. the logistic regression method [15]. On the other hand, the correlations between morphokinetic parameters are not a surprising phenomenon and indicate the existence of a certain cleavage pattern. The strongest correlation is observed for parameters which occur in short time intervals, for example $\mathrm{t} 5_{\text {med }}$ and t6 ${ }_{\text {med }}(R=0.85)$. It is obvious that the emergence of some abnormalities in embryo development influences subsequent events. The ensuing cleavages are related to each other and any deviation from the norm cannot be repaired immediately by the restoration of a normal pattern of further divisions. Similar, strong correlations are observed between relative and absolute morphokinetic parameters, especially if they occur at short time intervals. Only distant event correlations (for example t $2_{\text {med }}$ and t8int ${ }_{\text {med }}$ ) show no statistical significance.

Previous studies have demonstrated that incorporating morphological data normally used for embryo quality assessment considerably increases the predictive power of the created models. Further improvement can probably be effected by establishing more subtle relationships between parameters of different origin, not only morphological or 
morphokinetic, but possibly those from disparate disciplines such as genomics, proteomics or metabolomics. Data mining and artificial intelligence methods could meet this challenge and allow for the creation of comprehensive models of very strong predictive power.

\section{CONCLUSIONS}

Morphokinetic parameters are associated with embryo developmental and implantation potential and can be considered possible predictors of their quality. Their predictive power is greater when development to the blastocyst stage is analysed and slightly lower when a clinical pregnancy is predicted. The weakest association appears to exist between a biochemical pregnancy and morphokinetic data probably due to the fact that optimum conditions, which are sufficient for the implantation of even the weakest embryos, can be created. However, the implanted embryos' quality is verified at a later stage in the process of clinical pregnancy development. The determination of effective predictors cannot be limited to models based on singular parameters. The creation of more comprehensive models based on complex relationships between parameters will be far more beneficial to the pregnancy prediction process. Undoubtedly, a special role in complex model development can be played by data mining methods and artificial intelligence techniques, which may permit the extraction of information hidden in a large number of correlated parameters.

Morphokinetic parameters contain new information and can be used in the process of embryo developmental and implantation potential assessment. However, the creation of efficient pregnancy prediction models needs further studies which will be based on information included in all available parameters and utilise advanced biostatistical methods.

\section{REFERENCES}

1. Słomko Z. Ginekologia. 2nd ed. Wydawnictwo Lekarskie PZWL, 2008.

2. Milewski R, Milewska AJ, Czerniecki J, [et al.]. Analysis of the demographic profile of patients treated for infertility using assisted reproductive techniques in 2005-2010. Ginekol Pol. 2013, 84, 609-614.

3. Montag M, Liebenthron J, Köster M. Which morphological scoring system is relevant in human embryo development? Placenta. 2011, 32, 252-256.

4. Herrero J, Meseguer M. Selection of high potential embryos using time-lapse imaging: the era of morphokinetics. Fertil Steril. 2013, 99, 1030-1034.

5. Chamayou S, Patrizio P, Storaci G, [et al.]. The use of morphokinetic parameters to select all embryos with full capacity to implant. J Assist Reprod Genet. 2013, 30, 703-710.

6. Desai N, Ploskonka S, Goodman LR, [et al.]. Analysis of embryo morphokinetics, multinucleation and cleavage anomalies using continuous time-lapse monitoring in blastocyst transfer cycles. Reprod Biol Endocrinol. 2014, 12, 54.

7. Milewski R, Kuć P, Kuczyńska A, [et al.]. A predictive model for blastocyst formation based on morphokinetic parameters in time-lapse monitoring of embryo development. J Assist Reprod Genet. 2015, 32, 571-579.

8. Milewski R, Milewska AJ, Kuczyńska A, [et al.]. Do morphokinetic data sets inform pregnancy potential? J Assist Reprod Genet. 2016, 33, 357-365.

9. Cruz $\mathrm{M}, \mathrm{Garrido} N$, Herrero J, [et al.]. Timing of cell division in human cleavage-stage embryos is linked with blastocyst formation and quality. Reprod Biomed Online. 2012, 25, 371-381.

10. Cetinkaya M, Pirkevi C, Yelke H, [et al.]. Relative kinetic expressions defining cleavage synchronicity are better predictors of blastocyst formation and quality than absolute time points. J Assist Reprod Genet. 2015, 32, 27-35.

11. Lemmen JG, Agerholm I, Ziebe S. Kinetic markers of human embryo quality using time-lapse recordings of IVF/ICSI-fertilized oocytes. Reprod Biomed Online. 2008, 17, 385-391.

12. Meseguer $\mathrm{M}$, Herrero J, Tejera A, [et al.]. The use of morphokinetics as a predictor of embryo implantation. Hum Reprod. 2011, 26, 2658-2671.

13. Fréour T, Le Fleuter N, Lammers J, [et al.]. External validation of a time-lapse prediction model. Fertil Steril. 2015, 103, 917-922.

14. Aguilar J, Motato Y, Escribà MJ, [et al.]. The human first cell cycle: impact on implantation. Reprod Biomed Online. 2014, 28, 475-484.

15. Milewska AJ, Jankowska D, Citko D, [et al.]. The use of principal component analysis and logistic regression in prediction of infertility treatment outcome. Stud Logic Gramm Rhetor. 2014, 39, 7-23. 